\title{
Evolução do Programa de Triagem Neonatal em hospital de referência no Ceará: 11 anos de observação
}

\author{
Evolution of Neonatal Screening Program in a reference hospital in Ceará: \\ 11 years of observation
}

Daniela Cavalcanti e Silva Novais Carvalho'1, Tereza Callou Couto Macêdo¹, Magnollya Moreno², Francisco Winter dos Santos Figueiredo³, lara Bezerra Sales ${ }^{1}$, Juliana Christofolini ${ }^{4}$, Bianca Bianco ${ }^{4}$, Caio Parente Barbosa ${ }^{4}$, Denise Maria Christofolini ${ }^{4}$

${ }^{1}$ Faculdade de Medicina do ABC (FMABC) - Santo André (SP), Brasil.

${ }^{2}$ Universidade Federal do Cariri (UFCA) - Juazeiro do Norte (CE), Brasi

${ }^{3}$ Disciplina de Epidemiologia e Bioestatística, Departamento de Saúde Coletiva, FMABC - Santo André (SP), Brasil.

${ }^{4}$ Disciplina de Saúde Sexual e Reprodutiva e Genética de Populações, Departamento de Saúde Coletiva, FMABC - Santo André (SP), Brasil.

DOI: http://dx.doi.org/10.7322/abcshs.v42i3.933

\begin{abstract}
RESUMO
Introdução: O Programa Nacional de Triagem Neonatal (PNTN) tem como objetivo a detecção de doenças congênitas em fase pré-sintomática. Esse teste é realizado em todos os Estados do Brasil, porém com diferentes realidades quanto à incidência das doenças diagnosticadas devido à diversa origem étnica observada nacionalmente. O registro dos casos permite a elaboração de políticas públicas de prevenção e tratamento. Objetivo: Analisar a eficácia de um programa de triagem neonatal em um hospital de referência do Estado do Ceará ao longo dos últimos 11 anos. Métodos: Estudo transversal realizado a partir de prontuários de crianças nascidas entre os anos de 2002 e 2012 no referido hospital. Resultados: Dos 5.000 testes realizados, 2.893 prontuários completos foram incluídos no estudo (57,8\%). Desses casos, dois apresentaram fenilcetonúria e três, hipotireoidismo. Uma vez que quase a metade dos resultados dos testes não estava devidamente registrada no prontuário clínico dos pacientes, tornou-se inviável o cálculo de frequência da doença na população. Conclusão: A falha no registro dos dados do teste de triagem pode fazer com que falte aos gestores municipais e hospitalares o conhecimento sobre o número de casos da região, acarretando, consequentemente, falha nas estratégias de tratamento. Verifica-se a necessidade de organização de um banco de dados informatizado e de capacitação dos profissionais de saúde para o preenchimento dos dados do teste, para viabilizar o diagnóstico e o tratamento precoces, bem como permitir o delineamento do cenário real sobre a incidência das doenças diagnosticadas pelo "Teste do Pezinho" no Brasil.
\end{abstract}

Palavras-chave: anormalidades congênitas; triagem neonatal; doenças metabólicas.

\section{ABSTRACT}

Introduction: The National Neonatal Screening Program (PNTN) aims to detect pre-symptomatic congenital diseases. This test is performed across all States of Brazil with different realities concerning the incidence of diagnosed diseases due to the diverse national ethnicity observed. Accurate knowledge about diseases' incidence allows the elaboration of public policies for prevention and treatment. Objective: To evaluate the efficacy of neonatal screening program from a reference hospital in the State of Ceará for 11 years. Methods: Cross-sectional study based on medical records of children born between 2002 and 2012 in the referred hospital. Results: Of the 5,000 exams performed, 2,893 complete medical records (57.8\%) were included in the sample. Of these, two cases of phenylketonuria and three cases of hypothyroidism were observed. As almost half of the results had not been properly registered in patients' clinical records, the calculation of diseases frequency in this population was not reliable. Conclusion: Failure to register data of the screening test may impair knowledge acquisition of municipal and hospital managers when it comes to the number of cases in each region, consequently leading to a failure in treatment strategies. It is necessary to organize a digital database and to train health professionals to fill test result forms in order to enable early diagnosis and treatment, as well as to properly delineate a credible scenario of diseases' prevalence in Brazil.

Keywords: congenital abnormalities; neonatal screening; metabolic diseases. 


\section{INTRODUÇÃO}

A triagem neonatal é uma estratégia de saúde pública baseada no rastreamento de determinadas patologias na população neonatal (entre 0 e 30 dias de vida). Visa, principalmente, o diagnóstico e o início do tratamento precoces para evitar sequelas irreversíveis decorrentes das doenças triadas ${ }^{1,2}$.

No Brasil, o Programa Nacional de Triagem Neonatal (PNTN), conhecido como "Teste do Pezinho", foi implantado a partir da Portaria do Ministério da Saúde no 22, de janeiro de 1992, que foi atualizada na Portaria GM/MS no 822 , de 6 de junho de 2001, vigente até hoje. A portaria determinou a implantação, em todos os Estados e no Distrito Federal, de um Serviço de Referência em Triagem Neonatal (SRTN) e legitimou a obrigatoriedade da aplicação do teste a todos os recém-nascidos vivos ${ }^{3}$.

A implantação do PNTN nos Estados brasileiros é realizada em quatro fases, de acordo com as doenças avaliadas:

- Fase I: fenilcetonúria (PKU) e hipotireoidismo congênito (HC);

- Fase II: anemia falciforme e outras hemoglobinopatias;

- Fase III: fibrose cística (FC);

- Fase IV: deficiência de biotinidase e hiperplasia adrenal congênita.

O PNTN encontra-se em diferentes fases de implantação nos diversos Estados brasileiros ${ }^{3,4}$.

A coleta do exame é uma etapa fundamental do processo e deve ser realizada preferencialmente entre o $3^{\circ}$ e o $5^{\circ}$ dias após o nascimento da criança, por meio do colhimento, em papel-filtro, de algumas gotas de sangue do calcanhar da criança. O papel-filtro deve ser enviado ao SRTN do Estado onde a amostra foi recolhida. Após processadas as análises laboratoriais, o resultado volta para a unidade em que foi realizada a coleta ${ }^{5}$. Os resultados alterados são confirmados por meio de testes específicos em serviços de referência. Preconiza-se que o tempo entre o nascimento e o início do tratamento seja de até 14 dias $^{6}$.

A cobertura do programa corresponde à razão entre o número de amostras analisadas e o total de nascidos vivos. Porém, esse cálculo não consegue representar totalmente a realidade da cobertura, uma vez que nem todas as crianças têm seus exames realizados pelo Sistema Único de Saúde (SUS). As amostras de sangue coletadas encaminhadas para laboratórios particulares podem não ser computadas, já que não há obrigatoriedade de relatar às secretarias de saúde o número de exames realizados, podendo ocorrer subestimativa da cobertura ${ }^{7,8}$.

Somada a esses fatores, a falha no registro de resultados faz com que a prevalência das doenças triadas ainda seja desconhecida em parte dos Estados brasileiros.

O objetivo proposto neste artigo foi avaliar, ao longo de 11 anos, a eficácia de um programa de triagem neonatal em um hospital de referência do Estado do Ceará.

\section{MÉTODOS}

Trata-se de uma pesquisa transversal, com coleta de dados retrospectiva realizada por meio da análise de dados secundários obtidos a partir de livros de registro do "Teste do Pezinho" e das declarações de nascidos vivos utilizando a base de dados do Sistema de Notificação de Nascidos Vivos (SINASC). A pesquisa envolveu a população de crianças rastreadas pelo PNTN no Hospital e Maternidade São Vicente de Paulo, em Barbalha, Ceará, no período entre janeiro de 2002 (que corresponde à implantação do serviço de triagem neonatal) e dezembro de 2012, contemplando 11 anos de implantação do serviço de triagem neonatal no referido hospital. Na data final da coleta dos exames, o programa de triagem encontrava-se na fase II de implantação 9 .

Este estudo foi aprovado no Comitê de Ética em Pesquisa envolvendo seres humanos da Faculdade de Medicina do ABC/ Plataforma Brasil, com CAAE-17444213.900000082.

\section{RESULTADOS}

Dos cerca de 5.000 "Testes do Pezinho" ocorridos no hospital de referência entre 2002 e 2012, apenas 2.893 prontuários foram considerados para o estudo, por conterem o resultado do teste, verificando-se perda de $42 \%$ de registro dos resultados. Observou-se, no entanto, um número crescente de registros, iniciando em 2004, com apenas 26, e culminando em 2010 e 2011, com 1.321 e 1.166 resultados, respectivamente. Em 2012, novamente houve queda, com apenas 248 resultados registrados, devido à mudança na gestão hospitalar.

Em relação à presença de alterações rastreadas pelo "Teste do Pezinho", observou-se que $0,17 \%$ dos prontuários disponíveis, correspondendo a um total de cinco casos, possuíam alterações. As patologias observadas estão descritas a seguir: dois casos de fenilcetonúria $(0,07 \%$ da amostra) e três casos de hipotireoidismo $(0,1 \%$ da amostra). Não foi reportada consanguinidade direta entre os genitores dos casos de fenilcetonúria. No entanto, observou-se que as mães dos dois pacientes eram primas. Ressalta-se que em um caso observa-se a presença da fenilcetonúria associada à fenda labial; no outro caso houve associação entre hipotireoidismo, fenda palatina e lábio leporino. Os cinco casos de doenças metabólicas ocorreram nos anos de 2011 e 2012. A confirmação do diagnóstico de fenilcetonúria foi feita por meio da dosagem de fenilalanina e tirosina no soro e do hipotireoidismo congênito por meio da dosagem de T4 e TSH em amostra de sangue venoso, realizadas no Hospital Albert Sabin.

As características clínicas referentes às gestações e aos nascimentos de crianças com alterações no "Teste do Pezinho", bem como o tempo entre a coleta e o início do tratamento estão disponíveis na Tabela 1.

\section{DISCUSSÃO}

A maternidade-alvo deste estudo, localizada na região do Cariri, centro-sul do Estado do Ceará, é referência em alto risco na área materno-infantil. O hospital recebe atualmente pacientes provenientes de 20 municípios do Ceará, correspondendo a aproximadamente 1.000 nascidos por mês, entre SUS, convênios e atendimentos particulares.

Entretanto, nem todos os recém-nascidos realizam o "Teste do Pezinho" no hospital em que nasceram, uma vez que a indicação da 
coleta do teste, entre o $3^{\circ}$ e o $5^{\circ}$ dias de vida, muitas vezes não coincide com o período de internação do neonato, permitindo que o exame seja realizado no município de origem da gestante e/ou por meio de convênio médico, ou ainda coletas particulares, justificando, assim, o baixo número de exames realizados no referido hospital no período estudado em relação ao número de nascimentos. A falta de um prontuário eletrônico e de unificação dos dados dificulta o retorno de informações sobre os nascidos, demonstrado aqui pela perda de $42 \%$ do registro do resultado do "Teste do Pezinho". Ainda hoje, 2017, o registro dos resultados dos testes de triagem é feito de forma manual e descentralizada. $\mathrm{O}$ descaso com o registro das informações faz com que importantes dados sobre a saúde local sejam perdidos e que políticas públicas de atenção a esses pacientes deixem de ser aplicadas.

A pesquisa foi realizada com o levantamento de dados de 11 anos consecutivos. A ocorrência de doenças metabólicas diagnosticadas pelo "Teste do Pezinho" foi confirmada em cinco casos entre os 2.893 testes analisados $(0,17 \%$ da amostra). Deve-se ressaltar que não há registros de resultados com alterações no teste de triagem neonatal nos exames realizados entre 2002 e 2009. Uma possível explicação para esse fato é a baixa incidência da doença e o diminuto tamanho da amostra avaliada, que nesse período foi constituída por apenas 158 casos que continham a notificação do resultado do referido teste.

Estudos conduzidos no Brasil sobre a incidência de fenilcetonúria em amostras de triagem neonatal demonstraram resultados diversos nas diferentes regiões do país, como as prevalências de 1:1749 em Araraquara (SP), 1:8690 em Sergipe, 1:16.334 no Recôncavo Baiano, 1:19.409 em Ribeirão Preto (SP), 1:25.313 no Rio de Janeiro, 1:28.309 no Tocantins, 1:28.862 em Santa Catarina e 1:33.068 obtidos em um estudo realizado em Mato Grosso ${ }^{2,7,10-15}$. Essa importante diferença de número de casos observada entre os Estados pode ser devida à forma de registro e ao funcionamento dos serviços de triagem neonatal.

A incidência de $\mathrm{HC}$ na população mundial varia entre diferentes populações, com valores entre 1:20 e 1:6287 nascidos vivos, sendo as maiores prevalências verificadas em grupos étnicos específicos (México e Bielorrússia) ${ }^{16}$. No Brasil, os valores obtidos em diferentes estudos oscilam de 1:7.500 a 1:10.000 nascidos vivos ${ }^{2,14,17}$. No presente trabalho foi observada a prevalência de 1:964, estatística que provavelmente não reflete a realidade local, pela dificuldade de registro e pela falta de uniformização no Programa de Triagem Neonatal do Estado do Ceará. Não foram identificadas publicações anteriores a esta que refletissem a situação dessas doenças no Ceará.
De acordo com Nascimento et al. ${ }^{18}$, o período ideal para início do tratamento do HC deveria ser inferior a duas semanas, para que não haja sequelas neurológicas. Na presente avaliação, o período entre o nascimento da criança e o início do tratamento foi de até 18 dias nos casos de hipotireoidismo congênito e de até 28 dias nos casos de fenilcetonúria. Porém, todos os testes foram colhidos cinco dias após o nascimento da criança, e em um caso de fenilcetonúria, 12 dias após o nascimento. Há diversos fatores que contribuem para a demora na coleta do exame, como a falta de conscientização das famílias sobre a importância da realização do teste, os riscos associados à ocorrência de erros metabólicos para a saúde do nascido e o risco para novos nascimentos nas famílias. Ressalta-se também a falta de informação sobre a gratuidade do teste e do tratamento, se alguma das condições for detectada, por se tratar de um programa público de triagem neonatal implantado pelo Ministério da Saúde.

Entre os casos diagnosticados, foram observados um caso de hipotireoidismo associado à fenda labial e à palatina e um caso de fenilcetonúria associada à fenda palatina. Uma vez que a associação dessas condições pode indicar a ocorrência de uma síndrome genética, ressalta-se a importância da avaliação desses pacientes por um geneticista clínico, para a correta indicação do risco de recorrência nas famílias, que pode ser diferente da ocorrência isolada dessas condições.

A consulta de aconselhamento genético é uma prática de informação e comunicação para controle de riscos no processo reprodutivo, ou seja, risco de transmissão de uma característica própria ou familiar à prole ${ }^{19}$. Observou-se na presente pesquisa que, nos casos diagnosticados de fenilcetonúria, as mães eram primas e as crianças nasceram com intervalo maior do que 17 meses. Sabe-se que a confirmação diagnóstica de uma doença hereditária normalmente causa profundo impacto na família direta do bebê (pai, mãe e irmãos), a qual deve receber encaminhamento e acompanhamento clínico de uma equipe treinada. Os SRTNs devem ser compostos por uma equipe multidisciplinar que inclui médico pediatra, endocrinologista, geneticista, psicólogo, nutricionista e assistente social para dar suporte às famílias. Em um segundo momento, entretanto, a possibilidade de aconselhamento genético dever ser oferecida aos familiares portadores não manifestos das anomalias, visando à orientação sobre os riscos de recorrência para futuras gestações ${ }^{19}$.

Tanto a fenilcetonúria quanto o hipotireoidismo congênito são frequentemente assintomáticos nos primeiros meses de vida da criança $e$ tendem a agravar-se com o tempo, devido à ausência ou ao acúmulo

Tabela 1: Características dos recém-nascidos com alterações metabólicas no período entre 2002 a 2012 em Barbalha, Ceará

\begin{tabular}{|c|c|c|c|c|c|c|c|}
\hline \multirow{2}{*}{$\begin{array}{l}\text { Alteração congênita/ } \\
\text { metabólica }\end{array}$} & \multicolumn{7}{|c|}{ Características das crianças } \\
\hline & Sexo & $\begin{array}{l}\text { Semanas de } \\
\text { gestação }\end{array}$ & $\begin{array}{c}\text { Peso ao } \\
\text { nascer (g) }\end{array}$ & $\begin{array}{l}\text { Apgar } \\
1 / 5 \text { min }\end{array}$ & $\begin{array}{c}\text { Ano de } \\
\text { nascimento }\end{array}$ & $\begin{array}{c}\text { Tempo nascimento/ } \\
\text { coleta }\end{array}$ & $\begin{array}{l}\text { Tempo nascimento/ } \\
\text { tratamento }\end{array}$ \\
\hline Fenilcetonúria & $\mathrm{F}$ & $37-41$ & 3.160 & $8 / 9$ & 2011 & 7 dias & 22 dias \\
\hline Hipotireoidismo & $M$ & $37-41$ & 1.585 & $8 / 8$ & 2011 & 5 dias & 18 dias \\
\hline Hipotireoidismo & $\mathrm{F}$ & $37-41$ & 3.820 & $8 / 9$ & 2011 & 7 dias & 16 dias \\
\hline $\begin{array}{l}\text { Hipotireoidismo, fenda labial } \\
\text { e palatina }\end{array}$ & $\mathrm{F}$ & $32-36$ & 2.830 & $9 / 10$ & 2012 & 7 dias & 18 dias \\
\hline Fenilcetonúria e fenda labial & $M$ & $37-41$ & 2.860 & $8 / 9$ & 2012 & 12 dias & 28 dias \\
\hline
\end{tabular}

F: feminino; M: masculino.

Fonte: Secretaria Municipal de Saúde. Coordenadoria de Vigilância Epidemiológica. 
dos metabólitos não processados em pacientes não tratados. Assim, a detecção e o tratamento precoces são fundamentais nessas condições ${ }^{2}$.

As limitações deste estudo estão baseadas principalmente no baixo número de registro dos resultados dos testes realizados, impedindo, dessa forma, o cálculo preciso da prevalência das doenças rastreadas na região do Cariri. No entanto, não há outro relato sobre os resultados do teste de triagem neonatalno Ceará.

A detecção é a base para a triagem neonatal, para que possa haver o tratamento precoce das crianças afetadas. Para que seja cumprido, ressalta-se a importância do registro com todas as informações pertinentes à criança e de seu responsável para que a localização seja imediata. O SRTN deve contar com uma equipe de busca ativa, conforme elegantemente demonstrado por Kopacek et al., que avaliaram a evolução de um programa de triagem neonatal no Rio Grande do Sul ${ }^{20}$. Além disso, o conhecimento sobre a epidemiologia das doenças regionais também é de fundamental importância para os gestores em saúde e para o esclarecimento dos profissionais da atenção primária, secundária e terciária.

\section{REFERÊNCIAS}

1. Brasil. Ministério da Saúde [Internet]. Manual de normas técnicas e rotinas operacionais do Programa Nacional de Triagem Neonatal. Disponível em: http://bvsms.saude.gov.br/bvs/publicacoes/triagem_ neonatal.pdf. Acesso em: 14 jun. 2014.

2. Veslopi S, Marques H, Santos VF, Marane SSG, Chung MC, Santos JL. Análise das prevalências de doenças detectadas pelo programa nacional de triagem neonatal no município de Araraquara no ano de 2009. Rev Ciênc Farm Básica Apl. 2011;32(2):269-73.

3. Brasil. Ministério da Saúde [Internet]. Portaria MG/MS No 822/01 de 6 de junho de 2001. Disponível em: http://bvsms.saude.gov.br/ bvs/saudelegis/gm/2001/prt0822_06_06_2001.html. Acesso em: 08 mar. 2017.

4. Brasil. Ministério da Saúde [Internet]. Portaria MG/MS No 2829 de 14 de dezembro de 2012. Disponível em: http://bvsms.saude.gov. br/bvs/saudelegis/gm/2012/prt2829_14_12_2012.html

5. Abreu IS, Braguini WL. Triagem neonatal: o conhecimento materno em uma maternidade no interior do Paraná, Brasil. Rev Gaúcha Enferm. 2011;32(3):596-601.

http://dx.doi.org/10.1590/S1983-14472011000300023

6. Barone B, Lopes CLS, Tyszler LS, Amaral VB, Zarur RHC, Neto Paiva V, et al. Avaliação do valor de corte de TSH em amostras de filtro na triagem neonatal para diagnóstico de hipotireoidismo congênito no Programa "Primeiros Passos" - IEDE/RJ. Arq Bras Endocrinol Metab. 2013;57(1):57-61.

http://dx.doi.org/10.1590/S0004-27302013000100008

7. Amorim T, Boa-Sorte N, Leite MEQ, Acosta AX. Aspectos clínicos e demográficos da fenilcetonúria no Estado da Bahia. Rev Paul Pediatr. 2011;29(4):612-7.

http://dx.doi.org/10.1590/S0103-05822011000400022

8. Kopacek C, de Castro SM, Prado MJ, Silva CM, Beltrão LA, Spritzer PM. Neonatal screening for congenital adrenal hyperplasia in Southern Brazil: a population based study with 108,409 infants. BMC Pediatr. 2017;17(1):22.

https://dx.doi.org/10.1186/s12887-016-0772-x

9. Brasil. Ministério da Saúde. Programa Nacional de Triagem Neonatal. Nota informativa [Internet]. Brasília: Ministério da Saúde; 2012. Disponível em: http://triagemneonata|@saude.gov. br. Acesso em: 03 ago. 2017.

10. Ramalho AR, Ramalho RJ, Oliveira CR, Magalhães MM, Santos EG, Sarmento PM, et al. Evaluation of effectiveness and outcome of PKU screening and management in the State of Sergipe, Brazil. Arq Bras Endocrinol Metabol. 2014;58(1):62-7. http://dx.doi.org/10.1590/0004-2730000002885
11. Mendes LC, Santos TT, Bringel FA. Evolution of the neonatal screening program in the state of Tocantins. Arq Bras Endocrinol Metabol. 2013;57(2):112-9.

http://dx.doi.org/10.1590/S0004-27302013000200003

12. Stranieri I, Takano OA. Evaluation of the Neonatal Screening Program for congenital hypothyroidism and phenylketonuria in the State of Mato Grosso, Brazil. Arq Bras Endocrinol Metabol. 2009;53(4):446-52. http://dx.doi.org/10.1590/S0004-27302009000400010

13. Magalhães PKR, Turcato MF, Angulo IL, Maciel LMZ. Neonatal screening program at the university hospital of the Ribeirão Preto School of Medicine, São Paulo University, Brazil. Cad Saúde Pública. 2009;25(2):445-54. http://dx.doi.org/10.1590/S0102-311X2009000200023

14. Nunes AKC, Wacholz RG, Rover MRM, Souza LC. Prevalência de patologias detectadas pela triagem neonatal em Santa Catarina. Arq Bras Endocrinol Metab. 2013;57(5):360-7. http://dx.doi.org/10.1590/S0004-27302013000500005

15. Botler J, Camacho LAB, Cruz MM. Phenylketonuria, congenita hypothyroidism and haemoglobinopathies: public health issues for a Brazilian newborn screening program. Cad Saúde Pública. 2012;28(9):1623-31.

http://dx.doi.org/10.1590/S0102-311X2012000900002

16. Ford G, LaFranchi SH. Screening for congenital hypothyroidism: a worldwide view of strategies. Best Pract Res Clin Endocrinol Metab. 2014;28(2):175-87. http://dx.doi.org/10.1016/j.beem.2013.05.008

17. Costa SN, Oliveira EG, Boa Sorte N, Couto RD, Couto FD. Triagem neonatal para fenilcetonúria, hipotireoidismo congênito e hemoglobinopatias no recôncavo Baiano: Avaliação da cobertura em Cruz das Almas e Valença, Bahia. Rev Baiana Saúde Pública. 2012;36(3):831-43.

18. Nascimento ML, Pires MMS, Nassar SM, Ruhland L. Avaliação do programa de rastreamento neonatal para hipotireoidismo congênito da Secretaria de Estado da Saúde de Santa Catarina. Arq Bras Endocrinol Metabol. 2003;47(1):75-81. http://dx.doi.org/10.1590/S0004-27302003000100012

19. Corrêa MC, Guilam MC. Risk discourse and prenatal genetic counseling. Cad Saúde Pública. 2006;22(10):2141-9. http://dx.doi.org/10.1590/S0102-311X2006001000020

20. Kopacek C, Castro SM, Chapper M, Amorim LB, Lüdtke C, Vargas P. Evolução e funcionamento do Programa Nacional de Triagem Neonatal no Rio Grande do Sul de 2001 a 2015. Bol Cient Pediatr. 2015;4(3):70-4. 